\title{
Africa's burden of disease: The University of Cape Town Sub-Saharan Africa Centre for Chronic Disease
}

A Sub-Saharan Africa Centre for Chronic Disease has been established at the University of Cape Town (UCT). It aims to develop, evaluate and disseminate methods and programmes to prevent chronic disease, improve the quality of care for patients with chronic disease and reduce risk factors. It will focus on surveillance, advocacy and prevention.

Like the rest of South Africa, the Western Cape experiences a multiple burden of disease. These differ in geographical distribution and weight within the different districts and even the substructures of the Cape Town metropole. The identified and measured components of this burden are infectious diseases (tuberculosis (TB) and HIV), injuries (interpersonal violence and motor vehicle accidents), mental health disorders (related to alcohol and drugs such as tik) and chronic disease (diabetes, heart disease and cancer). This trend is mirrored nationally, although with differing weighting of these groupings.

The approach to the burden of disease is conceptualised within a framework that places its determinants in 'upstream' factors or 'root' causes that can be either societal or structural, or 'downstream' factors and more 'direct' causes that are either behavioural or biological. Upstream factors are typically poverty, homelessness, legislation and social exclusion, while downstream factors include poor nutrition, alcohol consumption, misuse of tobacco and infectious agents such as TB and HIV.

Measurement of the burden of disease provides a robust method of measuring the outcomes of health interventions, particularly if it is institutionalised, as in the Western Cape Department of Health, in co-operation with partners in the Department of Home Affairs, Statistics South Africa, the Medical Research Council and the universities. This has provided compelling data indicating, for instance, dramatic reduction of the infant mortality rate to 'pre-HIV levels' over a relatively short period of time following the introduction of an effective prevention of mother-to-child transmission programme in newborns. Similar surveillance envisaged for the Centre for Chronic Disease will provide data on the impact of interventions to reduce the burden of disease resulting from chronic disease across a wide front.

Cardiovascular disease, type 2 diabetes, cancer, chronic lung disease and depression are the major non-communicable

diseases reaching epidemic proportions in former Soviet bloc states and the low-income regions of the world. In the past 15 years South Africa has seen a similar trend in chronic disease prevalence, which forms an increasing component of the South African global burden of disease.

Mayosi et al. ${ }^{1}$ described South Africa as being 'in the midst of a profound health transition'. In their view South Africa has not derived the anticipated benefits from progressive health care policies, such as free primary health care, as a result of low-quality health care provision and uneven access to services. However, it is acknowledged that this is also because the upstream determinants of ill-health, such as poverty and inadequate education, lie beyond the reach of the health sector.

World Health Organization (WHO) estimates place the burden from non-communicable disease in South Africa as two to three times higher than that in developed countries. ${ }^{2}$ Almost counter-intuitively the burden of non-communicable disease is rising in rural communities, while it affects a disproportionate number of poor people in urban communities. Age-standardised mortality rates in Cape Town show a social gradient in which people living in the poor sub-district of Khayelitsha have 856 deaths per 100000 attributable to such diseases, compared with rates of 450 - 500 per 100000 in the wealthy northern and southern sub-districts of Cape Town. ${ }^{3}$

The consequences of these developments are far reaching with, for instance, an estimated increase in annual national disability grants to R4.2 billion by 2040 as a result of the increase in cardiovascular diseases. In South Africa the burden of chronic disease resulting from the exposure of initially healthy adult men to the mining industry is significant and ongoing. At another level the WHO estimates that there were 1 billion overweight adults worldwide in 2003 as a result of diets high in fat and sugar and less physically active populations. Many non-communicable diseases share common risk factors such as tobacco use, physical inactivity and an unhealthy diet that translate into cardiovascular disease, diabetes and cancer.

The Millennium Development Goals adopted by 189 countries in 2000 at the UN Millennium Summit target among others a reduction of poverty and malnutrition, increased empowerment of women and the promotion of education, all of which are determinants of health and play a role in the genesis of chronic disease.

In 2005 the WHO constituted the Commission on Social Determinants of Health to address the social factors leading to ill health and health inequities indicating a clear international focus on the 'upstream' factors. Improved health is a developmental outcome, but conversely health can be a driving force for development and economic enlistment. The Commission on Macroeconomics and Health asserts that if upstream factors are controlled in conjunction with improved health services to address downstream factors, impoverished families would enjoy longer, happier, more productive lives. ${ }^{4}$ They would have fewer children, who would then be better educated with improved economic prospects.

South Africa has been a global leader in developing and implementing appropriate legislation for tobacco control. 
The resulting actions are thought to have brought about the falling tobacco consumption in South Africa. While control of alcohol is dealt with in the Liquor Act, which was formulated mainly to reduce the socio-economic effect of alcohol misuse and promote a responsible and sustainable alcohol industry, its impact has been far less successful.

The burden of non-communicable disease on the African continent and in South Africa in particular continues to demonstrate the potential for a sustained rise. A significant investment in the health care system and in particular the primary health care system is therefore justified. Further innovative strategies and plans are needed to address the determinants of this disease burden.

Understanding the burden of disease and its components is fundamental to health planning, health decision making and health care delivery in both the public and the private sectors. The launch of the Sub-Saharan Centre for Chronic Disease at UCT is therefore important to the Western Cape Province, southern Africa and the broader continent. Situated in Africa, it will build capacity locally while strengthening ties with experts in the various fields internationally to the benefit of Africa in general.
This is an edited address by Craig Househam on the opening of the Bill Hoffenberg Conference Room and the launch of the SubSaharan Africa Centre for Chronic Disease at UCT on 4 November 2009.

\section{K C Househam}

Head: Department of Health

Provincial Government of the Western Cape

Cape Town

Corresponding author: K C Househam (khouseha@pgwc.gov.za)

\section{References}

1. Mayosi BM, Flisher AJ, Lalloo UG, Sitas F, Tollman SM, Bradshaw D. The burden of noncommunicable diseases in South Africa. Lancet 2009; 374: 934-947.

2. World Health Organization. The Global Burden of Disease: 2004 Update. Geneva: WHO, 2008.

3. Groenewald P, Bradshaw D, Daniels J, et al. Cause of Death and Premature Mortality in Cape Town, 2001 - 2006. Cape Town: South African Medical Research Council, 2008.

4. Feachem RGA. Commission on Macroeconomics and Health. Bull World Health Organ 2002; $80: 87$ 